\title{
Communication
}

\section{Precursor-Dependent Photocatalytic Activity of Carbon Dots}

\author{
Amadio Emanuele ${ }^{1, *(\mathbb{D})}$, Simone Cailotto ${ }^{1} \mathbb{D}$, Carlotta Campalani ${ }^{1}$, Lorenzo Branzi ${ }^{1}$, \\ Carlotta Raviola $^{2}{ }^{\mathbb{D}}$, Davide Ravelli ${ }^{2} \mathbb{D}$, Elti Cattaruzza ${ }^{1} \mathbb{D}$, Enrico Trave ${ }^{1}$, Alvise Benedetti ${ }^{1}{ }^{\mathbb{D}}$, \\ Maurizio Selva ${ }^{1}$ (i) and Alvise Perosa ${ }^{1}$ (D) \\ 1 Department of Molecular Sciences and Nanosystems, Ca' Foscari University of Venice, 30172 Venezia Mestre, \\ Italy; simone.cailotto@unive.it (S.C.); carlotta.campalani@unive.it (C.C.); lorenzo.branzi@unive.it (L.B.); \\ cattaruz@unive.it (E.C.); enrico.trave@unive.it (E.T.); benedett@unive.it (A.B.); selva@unive.it (M.S.); \\ alvise@unive.it (A.P.) \\ 2 PhotoGreen Lab, Department of Chemistry, University of Pavia, 27100 Pavia, Italy; \\ carlotta.raviola01@universitadipavia.it (C.R.); davide.ravelli@unipv.it (D.R.) \\ * Correspondence: emanuele.amadio@unive.it
}

Academic Editor: Davide Ravelli

Received: 28 November 2019; Accepted: 24 December 2019; Published: 26 December 2019

\begin{abstract}
This work systematically compares both structural features and photocatalytic performance of a series of graphitic and amorphous carbon dots (CDs) prepared in a bottom-up manner from fructose, glucose, and citric acid. We demonstrate that the carbon source and synthetic procedures diversely affect the structural and optical properties of the CDs, which in turn unpredictably influence their photo electron transfer ability. The latter was evaluated by studying the photo-reduction of methyl viologen. Overall, citric acid-CDs were found to provide the best photocatalytic performance followed by fructose- and glucose-CDs. However, while the graphitization of glucose- and citric acid-CDs favored the photo-reaction, a reverse structure-activity dependence was observed for fructose-CDs due to the formation of a large graphitic-like supramolecular assembly. This study highlights the complexity to design in advance photo-active bio-based carbon nanomaterials.
\end{abstract}

Keywords: carbon dots; photocatalysis; carbon source; biomass valorization

\section{Introduction}

Fluorescent carbon dots (CDs) have drawn considerable attention in a wide range of applications ranging from the biomedical [1-5] to energy-related [6,7] fields. Among them, CDs appeared to be a promising luminescent bio-based nanomaterial for photocatalytic applications. To date, the photocatalytic activity of CDs has been under-explored despite their remarkable light harvesting properties and excellent electron donor/acceptor capabilities. Moreover, most reported photo-systems of CDs are limited by the co-presence of precious metal complexes or enzymes as redox mediators [8-12]. The accurate design of CDs with tunable photo-electric properties is one of the major issues to face to achieve a real breakthrough in this field.

The luminescence properties of CDs are known to be structurally-dependent [13-16]. For instance, high photoluminescence properties were observed in amorphous CDs-owing to the presence of molecular-like fluorophores and heteroatom doping agents in their structures-synthesized under mild hydrothermal conditions [7-19], while graphitic-like CDs obtained via harsh pyrolytic treatments gave low luminescence. The graphitic core, indeed, acts as a quencher of the molecular fluorophore-derived photoluminescence via a fluorescence resonance energy transfer (FRET) mechanism [20].

Nonetheless, the degree of carbonization and the actual structure of CDs influence their photocatalytic behavior as well. In the photo-evolution of $\mathrm{H}_{2}$ through water splitting, different 
performances were observed using CDs—prepared from citric acid or aspartic acid-with different amorphous, graphitic, or graphitic nitrogen structures [10]. Moreover, the photoreduction of methyl viologen $\left(\mathrm{MV}^{2+}\right)$ turned out to be affected by the quinonic precursors employed in the multicomponent synthesis of amorphous nitrogen doped CDs [18]. In all these cases, a general and unique structure-photoactivity correlation could not be exhaustively highlighted due to the high variability of the synthetic methods, carbon sources, and dopants employed in the synthesis of these nanomaterials.

In connection to the wide-ranging interests of our group in developing biomass-derived platform chemicals [21-26], we recently reported a fundamental study on the photoreduction of methyl viologen using a set of citric acid-derived CDs, paving the way towards the rational design of carbon-nanoparticles for efficient photocatalytic organic transformations [27]. These studies demonstrated that the carbon-nitrogen source, the synthetic method, and the resulting structural properties strongly affect the electrochemical properties of CDs and thus their photo-reactivity. In general, the presence of nitrogen in the amorphous structure of CDs favors the photogeneration of reactive electrons, thus improving the photoreduction efficiency.

Herein, we propose to evaluate whether the carbon sources (citric acid, glucose, or fructose) influence structural features and photocatalytic efficiency. The aim is to explore new synthetic options to design CDs for photocatalytic applications. We hereby demonstrate that the carbon source, as well as the synthetic methodologies employed, strongly affect structural and optical properties of the CDs, thus resulting in different photocatalytic activities.

\section{Results}

\subsection{Synthesis and Characterization of the $C D s$}

In this work, six different sets of CDs were synthetized using two different preparation methods-hydrothermal and pyrolytic, which yield amorphous (a-) and graphitic (g-) CDs, respectively-and three sets of reagents: citric acid (Cit-), glucose (Glu-), and fructose (Fru-) (see Supplementary Materials for full synthesis details). Briefly, a-Cit-CDs, a-Glu-CDs, and a-Fru-CDs were hydrothermally synthesized, as previously reported [2,27], from glucose, fructose, and citric acid, respectively, to yield amorphous nanomaterial composed of light molecular-like organic compounds as well as poorly-defined carbonaceous substances. Graphitic g-Glu-CDs and g-Fru-CDs were synthesized for the first time via harsh thermolysis of neat glucose or fructose, respectively, following the already published procedure optimized for citric acid derived g-Cit-CDs [27]. The silent ${ }^{1} \mathrm{H}$ and ${ }^{13} \mathrm{C}\left\{{ }^{1} \mathrm{H}\right\}-\mathrm{NMR}$ (Supplementary Materials Figure S1 and Figure S2 for g-Glu-CDs and g-Fru-CDs, respectively, and [24] for g-Cit-CDs) likely excluded the presence of molecular species and suggested the presence of NMR-inactive nano-carbonaceous solids.

After having synthesized the nanomaterials, their morphologies and optical properties were fully compared by high-resolution transmission electron microscopy (HR-TEM), scanning electron microscopy (SEM), X-ray photoelectron spectroscopy (XPS), Fourier transform infrared spectroscopy (FT-IR), photoluminescence (PL), ultraviolet-visible spectroscopy (UV-Vis), and time-resolved PL measurements.

HR-TEM images (Figure 1) clearly demonstrate how the synthetic procedures affect the nanostructures: hydrothermal synthesis yielded low density and amorphous CDs, while pyrolysis rendered graphitic structures with well-defined shapes. a-Glu-CDs, a-Fru-CDs, and a-Cit-CDs were all poorly defined nanomaterials with very rarefied and irregular structures having a dimension ranging from 9 to $12 \mathrm{~nm}[2,27]$. On the other hand, g-Glu-CDs and g-Cit-CDs [27] were well-dispersed nanoparticles with a quasi-spherical shape about 7-9 and 2-7 nm in size. Surprisingly, both HR-TEM (Figure 1 and Supplementary Materials Figure S3 and Figure S4) and SEM (Figure 2, Supplementary Materials Figures S5-S8) images revealed the formation only for the g-Fru-CDs of supramolecular 
agglomerates of graphene-like crystalline sheets with multilayers nature having dimension between $0.5 \mu \mathrm{m}$ and $1.5 \mu \mathrm{m}$.
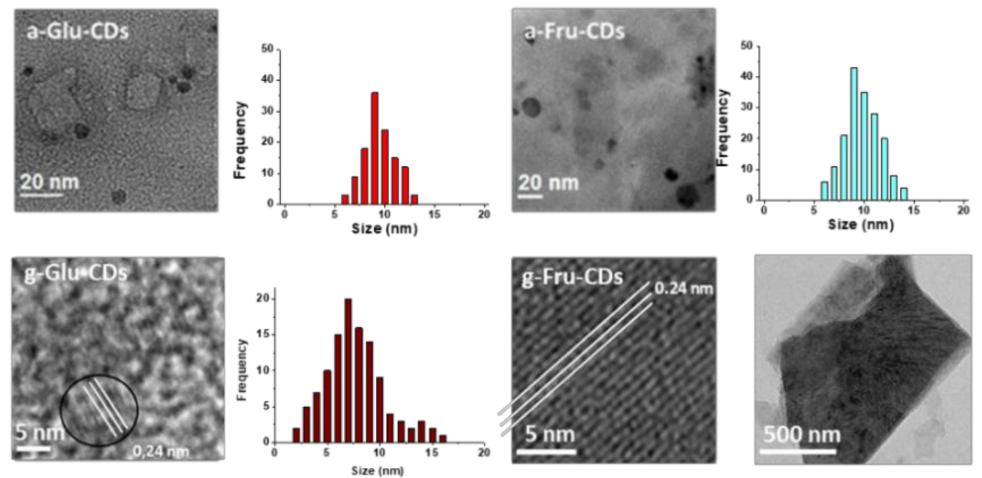
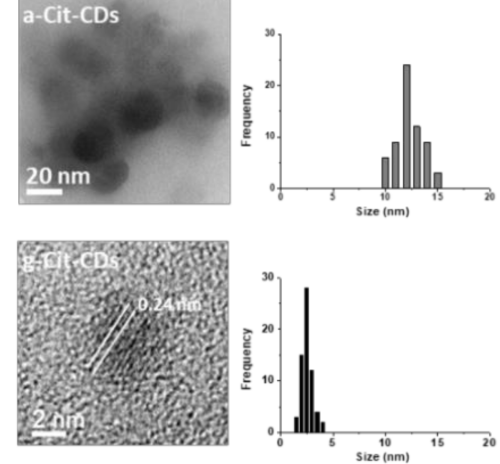

Figure 1. HR-TEM images of the amorphous and graphitic carbon dots (CDs).
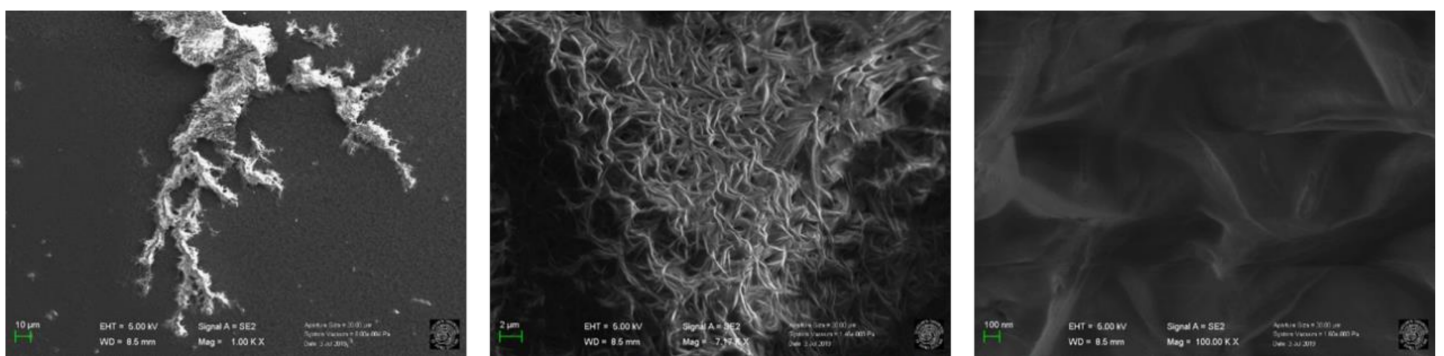

Figure 2. SEM images at increasing magnifications of g-Fru-CDs.

Substrate thermal decomposition influenced the structural differences of the graphitic CDs. By following the reactivity with ${ }^{1} \mathrm{H}-\mathrm{NMR}$ under pyrolysis condition (see Supplementary Materials for more details), citric acid and glucose were not fully converted after 60 min of heating while fructose quickly decomposed, leading to a silent spectrum as evidence of the reasonable formation of NMR inactive nano-structures. Citric acid decomposition pathways follow a known scheme, consisting first of a breakdown into small organic molecules (citraconic and itaconic anhydride) and then complete decomposition within 4 hours [27]. The monosaccharide sugars fructose and glucose follow instead different pathways: formic acid was mainly revealed during the thermal decomposition of fructose while acetic acid was detected from glucose [28]. Therefore, the final morphologies of the CDs may to a certain extent be derived by different decomposition pathways. Reasonably, the higher the reactivity of the carbon precursors, the easier and larger the formation of the nanoparticles.

Further evidence of the structural/compositional differences of the CDs was given by the XPS analysis. As shown in Figure 3, all samples displayed the presence of the three strong peaks in the $\mathrm{C} 1$ bands at 284.6, 286.2, and $288.6 \mathrm{eV}$ assigned to $\mathrm{C}=\mathrm{C}, \mathrm{C}-\mathrm{O}$, and $\mathrm{C}=\mathrm{O}$ functional groups, respectively, with, however, some differences. The $\mathrm{C}=\mathrm{C}$ signal was predominant for citric- and fructose-CDs while C-O was for glucose-CDs. Furthermore, the C-O band intensity, i.e., the oxygen content, markedly decreased after graphitization of citric acid and fructose as evidence of the formation of crystalline structures with predominantly graphitic $\mathrm{sp}^{2}$ carbon with a lower-with respect to the analogous glucose-like CDs-content of superficial carboxyl moieties.

However, no substantial differences were revealed by FT-IR analyses (Supplementary Materials Figure S12) since carboxylates-as primary surface CD functionalities-along with the formation of $\mathrm{C}\left(\mathrm{sp}^{2}\right)$ containing moieties were revealed in all cases. Indeed, a,g-Glu-CDs, a,g-Fru-CDs, and a,g-Cit-CDs all showed strong broad absorptions in the region $3500-3000 \mathrm{~cm}^{-1}$, weak signals at $2900-2800 \mathrm{~cm}^{-1}$, and additional stretching bands in the range of $1800-1600 \mathrm{~cm}^{-1}, 1600-1400 \mathrm{~cm}^{-1}$, 
and around $1200 \mathrm{~cm}^{-1}$, associated with the presence of $\mathrm{O}-\mathrm{H}, \mathrm{C}-\mathrm{H}, \mathrm{C}=\mathrm{O}, \mathrm{C}=\mathrm{C}$, and $\mathrm{C}-\mathrm{O}-\mathrm{C}$ functional groups, respectively.
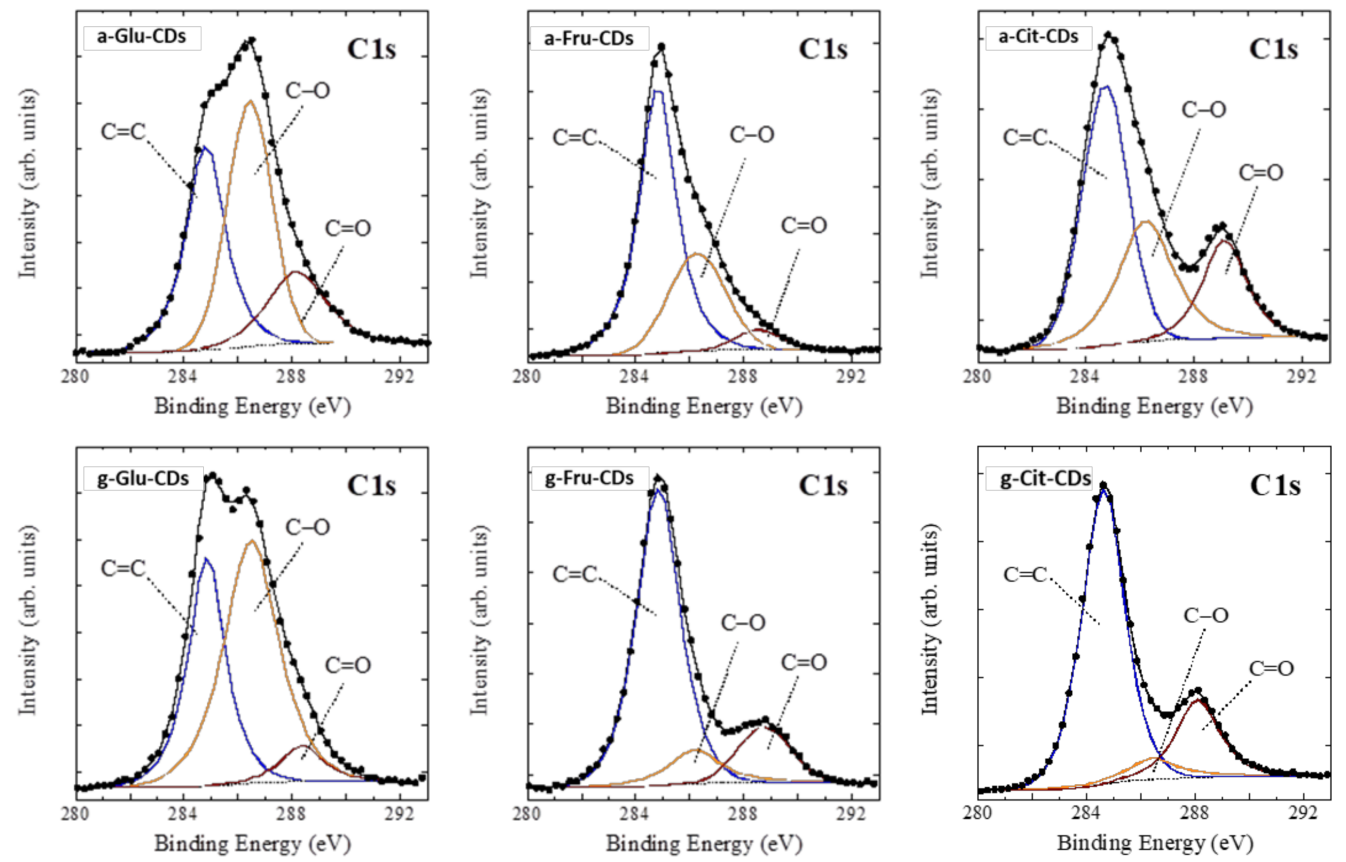

Figure 3. C1 XPS spectra of all the CDs samples. The binding energy (BE) was corrected for surface charging.

The photoluminescence (PL) of prepared CDs (Figure 4) showed a quasi-excitation-independent emission behavior with an emission peak centered at $450 \mathrm{~nm}$ that could be observed for both glucoseand fructose-CDs. The citric acid-derived nanomaterials exhibited instead a complex mixture of excitation dependent emission bands whose maximum ranged between 420 and $500 \mathrm{~nm}$. For g-Cit-CDs, an additional sharp band at $385 \mathrm{~nm}$ - slightly influenced by the excitation wavelength - was furthermore noticed due to the crystalline nano-core. The respective excitation spectra registered at different emission wavelength are shown in Supplementary Materials Figure S14.
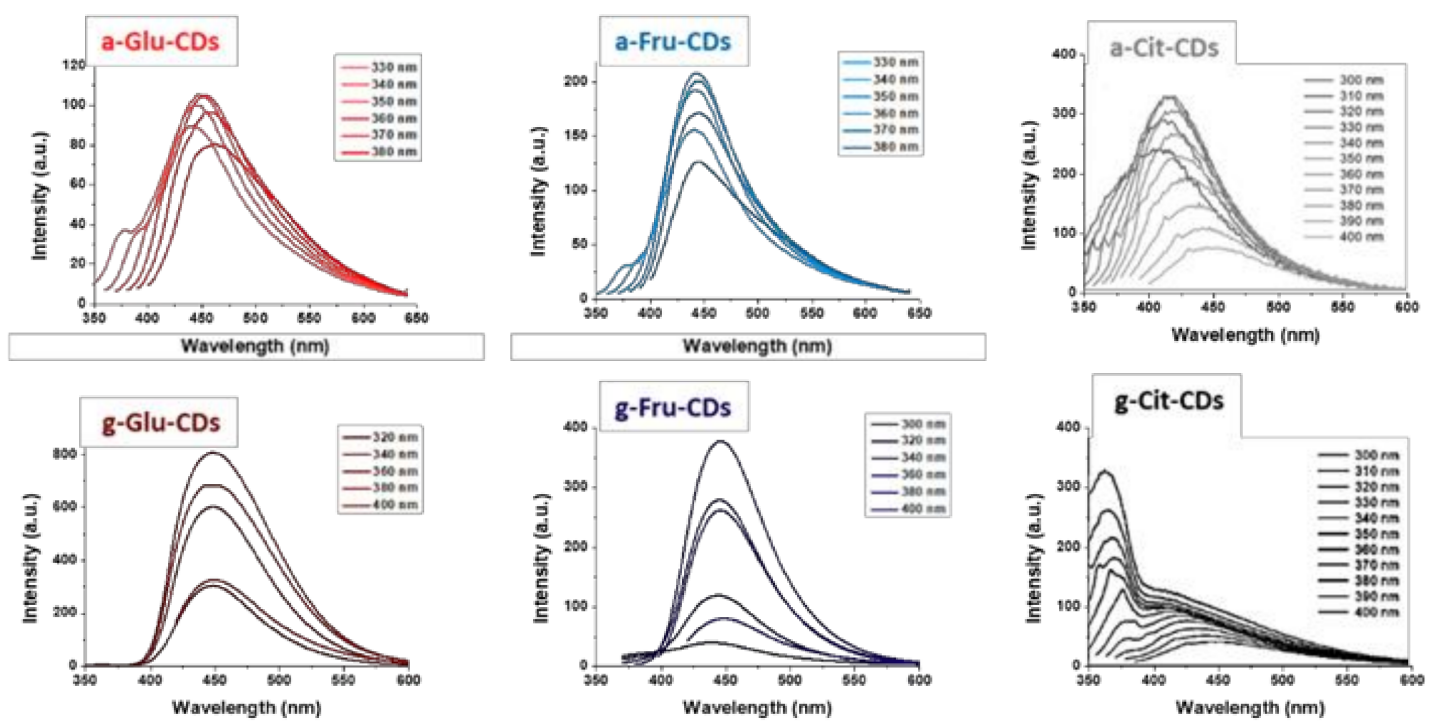

Figure 4. Photoluminescence (PL) spectra of amorphous and graphitic CDs. 
Lastly, UV-Vis spectra of all CDs (Supplementary Materials Figure S13) exhibited absorption bands across the near-UV and tailing in the visible region. In detail, g-Cit-CDs showed relatively simple spectra having a weak absorption band at $365 \mathrm{~nm}$, ascribed to $\mathrm{n}-\pi^{*}$ transition due to defects states [27], and a strong one at $220 \mathrm{~nm}$ due to the $\pi-\pi^{*}$ transition of the aromatic domains. a,g-Fru-CDs and a,g-Glu-CDs and a-Cit-CDs revealed instead the presence of three defined absorption regions at 220-225, 260-280, and $365-370 \mathrm{~nm}$ associated with the $\pi-\pi^{*}$ transition of $\mathrm{sp}^{2} \mathrm{C}=\mathrm{C}$ conjugated systems, $\mathrm{n}-\pi^{*}$ transitions of carbonyl groups, and surface state transitions, respectively. The photoluminescence quantum yields (QYs, based on quinine sulphate) of a-Cit-CDs and g-Cit-CDs were $1.0 \%$ and $1.2 \%$, respectively, and a-Glu-CDs and g-Glu-CDs gave slightly higher values of $1.8 \%$ and $2.3 \%$, respectively. Low QY values of $0.3 \%$ and $0.7 \%$, respectively, were instead measured for a-Fru-CDs and g-Fru-CDs [2,27]. The mass extinction coefficients $(\varepsilon)$ for CDs at $365 \mathrm{~nm}$ are reported in Table 1. Both g-Fru-CDs and g-Cit-CDs with graphitic cores showed significantly increased absorption (1.2 and 4 times, respectively) in comparison to the corresponding amorphous a-Fru-CDs and a-Cit-CDs. Conversely, a-Glu-CDs exhibited an improved absorption over graphitic ones (up to 2.7 times). Similar behavior was observed for the lifetimes of excited states (Table 1); the graphitic domain of g-Cit-CDs and g-Fru-CDs resulted in longer lifetimes in comparison with the analogous amorphous (from 3.3 and 4.4 for the amorphous to 5.4 and $5.9 \mathrm{~ns}$ for the graphitic, respectively). On the other hand, g-Glu-CDs (2.4 ns) possessed a lower lifetime compare to a-Glu-CDs (4.6 ns). All the CDs exhibited a PL decay characterized by a multiexponential behavior, whose parameters are reported in Supplementary Materials Table S1 and Figure 5.

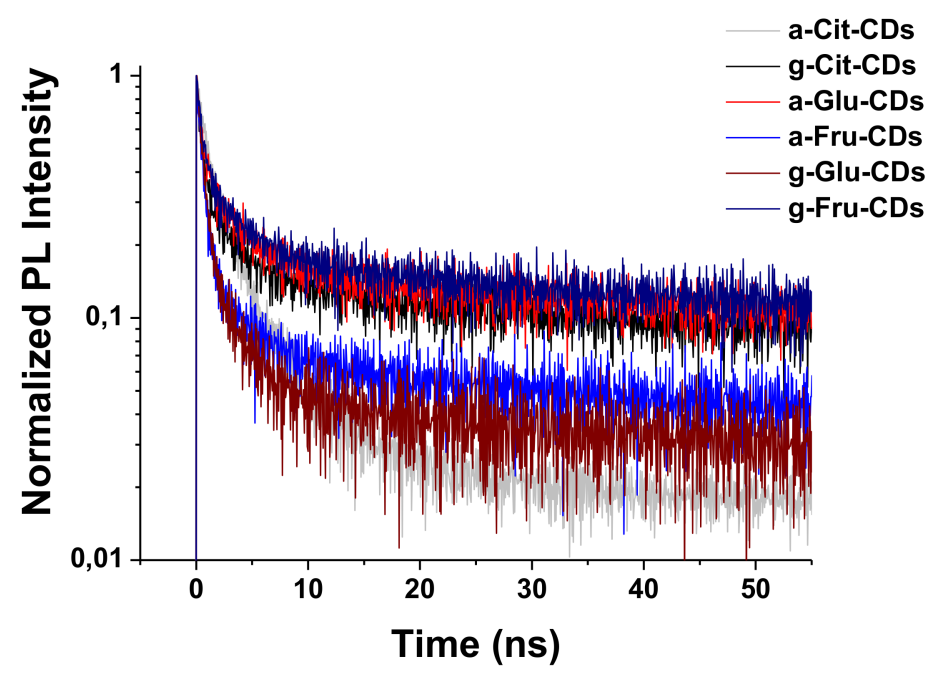

Figure 5. Time-resolved photoluminescent (PL) measurements of CDs.

Table 1. Mass extinction coefficients $(\varepsilon)$ for CDs calculated at $365 \mathrm{~nm}$ and lifetime of the excited states $(\tau)$.

\begin{tabular}{ccc}
\hline Entry & $\mathcal{\varepsilon}\left(\mathbf{L} \times \mathbf{g} \mathbf{-}^{\mathbf{1}} \times \mathbf{c m}^{\mathbf{- 1}}\right)$ & $\boldsymbol{\tau} \mathbf{~ ( n s )}$ \\
\hline a-Glu-CDs & 1.71 & 4.6 \\
g-Glu-CDs & 0.63 & 2.4 \\
a-Fru-CDs & 1.63 & 4.4 \\
g-Fru-CDs & 2.01 & 5.9 \\
a-Cit-CDs & $1.16[27]$ & 3.3 \\
g-Cit-CDs & $4.68[27]$ & 5.4 \\
\hline
\end{tabular}

\subsection{Photocatalytic Experiment}

To investigate the relationship between the structural features and their photo-activity, all CDs were subsequently employed as photo-redox catalysts in the single electron photoreduction of methyl 
viologen $\mathrm{MV}^{2+}\left(-0.45 \mathrm{~V}\right.$ vs. NHE) [29] to its mono-reduced species $\left(\mathrm{MV}^{\bullet+}\right)$. The photoreductions were carried out in aqueous media in the presence of ethylenediaminetetraacetate (EDTA) as a sacrificial electron donor and under LED light irradiation $(365 \mathrm{~nm})$ using a concentration of each CD normalized for absorption (0.5 a.u.). The lack of activity in the absence of light and CDs (Figure S15) indicated the primary role of the photo-excited CD-states in the photoinduced electron transfer (PET) reactivity.

As shown in Figure 6 and Table 2, the PET ability was found to be strongly dependent on the carbon precursor. Citric acid-derived CDs were the most photoactive nano-systems, followed by fructose and glucose analogues. The amorphous or graphitic structures influenced CD reactivity, further supporting the unpredictability of $C D$ photochemical behavior. In this case, the graphitization of citric acid and, surprisingly, also glucose-derived CDs enhanced their photocatalytic performance ca. 1.5 and 1.6 times, respectively, from an initial rate of 3.45 to $5.06 \times 10^{-8} \mathrm{M} \times \mathrm{s}^{-1}$ for a,g-Cit-CDs and from 0.65 to $1.07 \times 10^{-8} \mathrm{M} \times \mathrm{s}^{-1}$ for a,g-Glu-CDs (Table 2 entries 5,6, and 1,2). Nonetheless, a-Glu-CDs also exhibited an induction time of $10 \mathrm{~min}$. The opposite trend was instead observed for a,g-Fru-CDs, with the amorphous material being 6.7 time more reactive-from an initial rate of 4.37 to $0.67 \times 10^{-8} \mathrm{M} \times \mathrm{s}^{-1}$ - than the graphitic counterpart (Table 2 entries 3,4). The reason for this inversion was that the reactive performances reasonably rely on the formation of the large graphitic materials and supramolecular aggregates, which may affect the PET efficiency. Overall, the results herein reported highlight the complexity in assessing PET and in designing CDs-based photocatalytic organic applications.
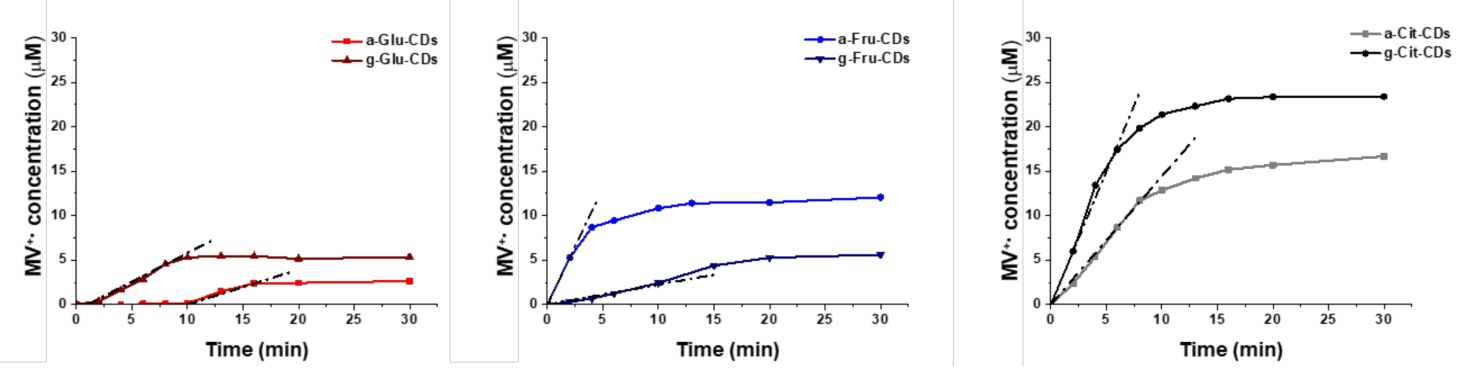

Figure 6. Reaction kinetics of formation of $\mathrm{MV}^{\bullet+}$ using CDs as photo-redox catalysts. Reaction conditions: $\mathrm{MV}^{2+} 60 \mu \mathrm{M}$, EDTA $0.1 \mathrm{M}, \lambda_{\text {exc }}$ : $365 \mathrm{~nm}$ with an absorbance-normalized amount of CDs and water as solvent (see Supplementary Materials for more details).

Table 2. Photocatalytic performance of the tested CDs.

\begin{tabular}{cccc}
\hline Entry & CDs & $\boldsymbol{v}_{\mathbf{0}} \times \mathbf{1 0}^{\mathbf{- 8}} \mathbf{( \mathbf { M } \times \mathbf { s } ^ { - \mathbf { 1 } } )}$ & Relative Rate \\
\hline 1 & a-Glu-CDs & $0.65^{\mathrm{a}}$ & 1 \\
2 & g-Glu-CDs & 1.07 & 1.6 \\
3 & a-Fru-CDs & 4.37 & 6.7 \\
4 & g-Fru-CDs & 0.67 & 1 \\
5 & a-Cit-CDs & $3.45[27]$ & 5.3 \\
6 & g-Cit-CDs & $5.06[27]$ & 7.8 \\
\hline
\end{tabular}

For reaction conditions, see Figure 6a. Calculated without considering the induction time.

\section{Conclusions}

In conclusion, the photocatalytic properties of monosaccharide-derived CDs were tested in the single electron transfer reaction towards $\mathrm{MV}^{2+}$. We demonstrate how the choice of the carbon precursors and its morphology can significantly influence the photo-redox behavior of the resulting materials.

The first significant finding herein described is that the hydrothermal treatment of citric acid, glucose, or fructose produced amorphous nanoaggregates containing molecular-like compounds, whereas pyrolysis formed extended graphene-like carbonaceous cores. Moreover, it was demonstrated 
that the higher the reactivity towards the decomposition/transformation of the carbon precursors, the easier and larger the formation of the nanoparticles was. In this regard, g-Fru-CDs resulted as a supramolecular agglomerate of graphene-like crystalline sheets with a multilayered nature.

It was also shown that synthetic methods and starting precursors strictly affected the photo and optical properties of the CDs as well. Citric acid, as a carbon source, yielded the best performing photoactive nanomaterials followed by fructose and glucose derivatives. Another important finding relied on the effect of the graphitization of the carbon core of CDs on their PET activity. The morphology of the carbon-dots induced either a positive or negative photo-reactivity trend, which in turn was influenced by the carbon-precursor employed. For fructose-CDs, the amorphous structure provided optimal results in terms of initial rates; likewise, the graphitic nanoparticles were more active when formed by citric acid and glucose.

Overall, these results provide important insights into carbon source/structure/activity relationships and may pave the way for a more rational development of carbon-derived nano-photocatalysts for organic synthesis.

Supplementary Materials: The following are available online at http://www.mdpi.com/1420-3049/25/1/101/s1: Materials and methods, synthesis and characterization of CDs, thermal decomposition studies, TEM and SEM images, XPS, FT-IR, UV spectra, time-resolved photoluminescence, and photocatalytic experiments.

Author Contributions: Conceptualization, A.E. and A.P.; validation, A.E., D.R., A.B., M.S. and A.P.; investigation, S.C., C.C., L.B., C.R., E.C. and E.T.; writing-original draft preparation, A.E., S.C. and A.P.; writing-review and editing, A.E., S.C., D.R. and A.P.; supervision, A.E. and A.P.; funding acquisition, D.R., M.S. and A.P. All authors have read and agreed to the published version of the manuscript.

Funding: This research received no external funding.

Acknowledgments: E.A. acknowledges the European Social Fund for a post-doctoral scholarship.

Conflicts of Interest: The authors declare no conflict of interest.

\section{References}

1. Chen, B.; Li, F.; Li, S.; Weng, W.; Guo, H.; Guo, T.; Zhang, X.; Chen, Y.; Huang, T.; Hong, X.; et al. Large scale synthesis of photoluminescent carbon nanodots and their application for bioimaging. Nanoscale 2013, 5, 1967. [CrossRef] [PubMed]

2. Cailotto, S.; Amadio, E.; Facchin, M.; Selva, M.; Pontoglio, E.; Rizzolio, F.; Riello, P.; Toffoli, G.; Benedetti, A.; Perosa, A. Carbon Dots from Sugars and Ascorbic Acid: Role of the Precursors on Morphology, Properties, Toxicity, and Drug Uptake. ACS Med. Chem. Lett. 2018, 9, 832-837. [CrossRef] [PubMed]

3. Pardo, J.; Peng, Z.; Leblanc, R.M. Cancer Targeting and Drug Delivery Using Carbon-Based Quantum Dots and Nanotubes. Molecules 2018, 23, 378. [CrossRef] [PubMed]

4. Huang, S.-W.; Lin, Y.-F.; Li, Y.-X.; Hu, C.-C.; Chiu, T.-C. Synthesis of Fluorescent Carbon Dots as Selective and Sensitive Probes for Cupric Ions and Cell Imaging. Molecules 2019, 24, 1785. [CrossRef] [PubMed]

5. Garg, B.; Bisht, T. Carbon Nanodots as Peroxidase Nanozymes for Biosensing. Molecules 2016, $21,1653$. [CrossRef]

6. Tuerhong, M.; Xu, Y.; Yin, X.-B. Review on Carbon Dots and Their Applications. Chin. J. Anal. Chem. 2017, 45, 139-150. [CrossRef]

7. Chandra, S.; Patra, P.; Pathan, S.H.; Roy, S.; Mitra, S.; Layek, A.; Bhar, R.; Pramanik, P.; Goswami, A. Luminescent S-doped carbon dots: An emergent architecture for multimodal applications. J. Mater. Chem. B 2013, 1, 2375. [CrossRef]

8. Hutton, G.A.M.; Martindale, B.C.M.; Reisner, E. Carbon dots as photosensitisers for solar-driven catalysis. Chem. Soc. Rev. 2017, 46, 6111-6123. [CrossRef]

9. Martindale, B.C.M.; Hutton, G.A.M.; Caputo, C.A.; Reisner, E. Solar Hydrogen Production Using Carbon Quantum Dots and a Molecular Nickel Catalyst. J. Am. Chem. Soc. 2015, 137, 6018-6025. [CrossRef]

10. Martindale, B.C.M.; Hutton, G.A.M.; Caputo, C.A.; Prantl, S.; Godin, R.; Durrant, J.R.; Reisner, E. Enhancing Light Absorption and Charge Transfer Efficiency in Carbon Dots through Graphitization and Core Nitrogen Doping. Angew. Chem. Int. Ed. 2017, 56, 6459-6463. [CrossRef] 
11. Han, M.; Zhu, S.; Lu, S.; Song, Y.; Feng, T.; Tao, S.; Liu, J.; Yang, B. Recent progress on the photocatalysis of carbon dots: Classification, mechanism and applications. Nano Today 2018, 19, 201-218. [CrossRef]

12. Wang, R.; Lu, K.-Q.; Tang, Z.-R.; Xu, Y.-J. Recent progress in carbon quantum dots: Synthesis, properties and applications in photocatalysis. J. Mater. Chem. A 2017, 5, 3717-3734. [CrossRef]

13. Shi, L.; Yang, J.; Zeng, H.B.; Chen, Y.M.; Wu, C.; Osada, Y.; Zhang, Q.Q. Carbon dots with high fluorescent quantum yield: The fluorescence originates from organic fluorophores. Nanoscale 2016, 8, 14374-14378. [CrossRef] [PubMed]

14. Wang, S.; Chen, Z.-G.; Cole, I.; Li, Q. Structural evolution of graphene quantum dots during thermal decomposition of citric acid and the corresponding photoluminescence. Carbon 2015, 82, 304-313. [CrossRef]

15. Krysmann, M.J.; Kelarakis, A.; Dallas, P.; Giannelis, E.P. Formation Mechanism of Carbogenic Nanoparticles with Dual Photoluminescence Emission. J. Am. Chem. Soc. 2012, 134, 747-750. [CrossRef] [PubMed]

16. Song, Y.; Zhu, S.; Zhang, S.; Fu, Y.; Wang, L.; Zhao, X.; Yang, B. Investigation from chemical structure to photoluminescent mechanism: A type of carbon dots from the pyrolysis of citric acid and an amine. J. Mater. Chem. C 2015, 3, 5976-5984. [CrossRef]

17. Gharat, P.M.; Chethodil, J.M.; Srivastava, A.P.; Praseetha, P.K.; Pal, H.; Choudhury, S.D. An insight into the molecular and surface state photoluminescence of carbon dots revealed through solvent-induced modulations in their excitation wavelength dependent emission properties. Photochem. Photobiol. Sci. 2019, 18, 110-119. [CrossRef]

18. Ren, W.-J.; Bai, J.-J.; Zhao, Y.-L.; Wang, Y.-L.; Liu, F.; Li, Z.-Z. One-pot synthesis of carbon dots co-doped with $\mathrm{N}$ and S: High quantum yield governed by molecular state and fluorescence detection of Ag+. Mol. Phys. 2019, 117, 2500-2510. [CrossRef]

19. Rodriguez-Padron, D.; Algarra, M.; Tarelho, L.A.C.; Frade, J.R.; Franco, A.; De Miguel, G.; Jimenez, J.; Rodríguez-Castellón, E.; Luque, R. Catalyzed Microwave-Assisted Preparation of Carbon Quantum Dots from Lignocellulosic Residues. ACS Sustain. Chem. Eng. 2018, 6, 7200-7205. [CrossRef]

20. Fang, Q.; Dong, Y.; Chen, Y.; Lu, C.-H.; Chi, Y.; Yang, H.-H.; Yu, T. Luminescence origin of carbon based dots obtained from citric acid and amino group-containing molecules. Carbon 2017, 118, 319-326. [CrossRef]

21. Rigodanza, F.; Đorđević, L.; Arcudi, F.; Prato, M.; Dordevich, L. Customizing the Electrochemical Properties of Carbon Nanodots by Using Quinones in Bottom-Up Synthesis. Angew. Chem. Int. Ed. 2018, 57, 5062-5067. [CrossRef] [PubMed]

22. Calmanti, R.; Galvan, M.; Amadio, E.; Perosa, A.; Selva, M. High-Temperature Batch and Continuous-Flow Transesterification of Alkyl and Enol Esters with Glycerol and Its Acetal Derivatives. ACS Sustain. Chem. Eng. 2018, 6, 3964-3973. [CrossRef]

23. Amadio, E.; González-Fabra, J.; Carraro, D.; Denis, W.; Gjoka, B.; Zonta, C.; Bartik, K.; Cavani, F.; Solmi, S.; Bo, C.; et al. Efficient Vanadium-Catalyzed Aerobic C-C Bond Oxidative Cleavage of Vicinal Diols. Adv. Synth. Catal. 2018, 360, 3286-3296. [CrossRef]

24. Amadio, E.; Di Lorenzo, R.; Zonta, C.; Licini, G.M. Vanadium catalyzed aerobic carbon-carbon cleavage. Coord. Chem. Rev. 2015, 301, 147-162. [CrossRef]

25. Fiorani, G.; Perosa, A.; Selva, M. Dimethyl carbonate: A versatile reagent for a sustainable valorization of renewables. Green Chem. 2018, 20, 288-322. [CrossRef]

26. Cattelan, L.; Yuen, A.K.L.; Lui, M.Y.; Masters, A.F.; Selva, M.; Perosa, A.; Maschmeyer, T. Renewable Aromatics from Kraft Lignin with Molybdenum-Based Catalysts. Chem. Cat. Chem. 2017, 9, 2717-2726. [CrossRef]

27. Cailotto, S.; Mazzaro, R.; Enrichi, F.; Vomiero, A.; Selva, M.; Cattaruzza, E.; Cristofori, D.; Amadio, E.; Perosa, A. Design of Carbon Dots for Metal-free Photoredox Catalysis. ACS Appl. Mater. Interfaces 2018, 10, 40560-40567. [CrossRef]

28. Huang, X.; Duan, H.; Barringer, S.A. Effects of buffer and temperature on formation of furan, acetic acid and formic acid from carbohydrate model systems. LWT 2011, 44, 1761-1765. [CrossRef] 
29. Striepe, L.; Baumgartner, T. Viologens and Their Application as Functional Materials. Chem. Eur. J. 2017, 23, 16924-16940. [CrossRef]

Sample Availability: Samples of the compounds a,g-Cit-CDs, a,g-Fru-CDs and a,g-Glu-CDs are available from the authors.

(C) 2019 by the authors. Licensee MDPI, Basel, Switzerland. This article is an open access article distributed under the terms and conditions of the Creative Commons Attribution (CC BY) license (http://creativecommons.org/licenses/by/4.0/). 